Research Article

Chen Lei*, Liu Gang, Lu Xingguo, Xu Minghai, and Tang Yuannan

\title{
Modeling the initial mechanical response and yielding behavior of gelled crude oil
}

https://doi.org/10.1515/phys-2018-0041

Received Sep 14, 2016; accepted Feb 24, 2017

\begin{abstract}
The initial mechanical response and yielding behavior of gelled crude oil under constant shear rate conditions were investigated. By putting the Maxwell mechanical analog and a special dashpot in parallel, a quasiJeffreys model was obtained. The kinetic equation of the structural parameter in the Houska model was simplified reasonably so that a simplified constitutive equation of the special dashpot was expressed. By introducing a damage factor into the constitutive equation of the special dashpot and the Maxwell mechanical analog, we established a constitutive equation of the quasi-Jeffreys model. Rheological tests of gelled crude oil were conducted by imposing constant shear rates and the relationship between the shear stress and shear strain under different shear rates was plotted. It is found that the constitutive equation can fit the experimental data well under a wide range of shear rates. Based on the fitted parameters in the quasi-Jeffreys model, the shear stress changing rules of the Maxwell mechanical analog and the special dashpot were calculated and analyzed. It is found that the critical yield strain and the corresponding shear strain where shear stress of the Maxwell analog is the maximum change slightly under different shear rates. And then a critical damage softening strain which is irrelevant to the shearing conditions was put forward to describe the yielding behavior of gelled crude oil.
\end{abstract}

Keywords: gelled crude oil; initial mechanical response; yielding; damage variable; critical damage softening strain

PACS: 83.60.Pq, 83.60.La, 83.60.Df

\footnotetext{
^Corresponding Author: Chen Lei: College of Pipeline and Civil Engineering, China University of Petroleum (East China), Qingdao 266580, China; Shandong Province Key Laboratory of Oil gas Storage and Transportation Safety, China University of Petroleum (East China), Qingdao 266580, China; Email: leo@upc.edu.cn,

Tel.: +86 13465815606

Liu Gang, Lu Xingguo, Xu Minghai, Tang Yuannan: College of Pipeline and Civil Engineering, China University of Petroleum (East China), Qingdao 266580, China; Shandong Province Key Laboratory
}

\section{Introduction}

The waxy crude oil needs to be heated usually when transported in pipelines. However, these pipelines may inevitably be shutdown due to transport plans or accidents. The temperature of heated oil is usually higher than the environment temperature and drops after the shutdown of pipelines. Waxy crystals will dissipate and build up gelled structure which increase the oil viscosity and impair the restart of pipe flow. The rheological properties of gelled crude oil are quite crucial for calculating the pipeline restart properties. Elastic-limit stress, static stress and dynamic yield stress were proposed by Cheng Chang to describe the yielding property of gelled crude oil during the pipeline restart [1]. The Houska thixotropic model was selected to represent the flow property of gelled crude oil in the 1.5D start-up model proposed by Wachs, A. [2]. When investigating the pressure wave velocity during the initial startup of gelled crude oil pipeline, a pure thixotropic model was chosen for calculating, and the initial shear stress was regarded as the yield stress by Zhang, G. [3]. The shear stress growth process before yielding was not considered when investigating the pipeline restart in the above literature. But Negrão, C.O.R. [4] regarded that the initial stress growth before yielding should be considered when exploring the restart properties of drilling fluid pipeline numerically. Similar to the drilling fluid, gelled crude oils always have a significant shear stress growth before yielding [5]. Based on the experimental data in literature [3], we can find that the time period needed for the start-up wave passing through the whole pipe is quite short. During such a short time period, the thixotropic process may be insignificant but the stress response process before yielding plays a leading role. So, when investigating the restart of gelled crude oil pipeline, the initial mechanical response and the yielding properties of gelled crude oil should not be ignored [6].

of Oil gas Storage and Transportation Safety, China University of Petroleum (East China), Qingdao 266580, China 
For the yielding properties of gelled crude oil, scholars have carried out many research studies. Among the properties, the yield stress is an important parameter to describe the yielding process. However, the yield stress of gelled crude oil is correlated significantly with the shear conditions, such as the loading rate of shear stress [7]. Through large amount of experiments, Li, C.X. [8] and Hou, L. [9] found out that the critical yield strain of gelled crude oil under different loading conditions remains unchanged and the shear strain was chosen as the index to measure the yielding behavior of gelled crude oil. But, just relying on the yield strain of gelled crude oil cannot figure out the restart process of pipeline. Thus, scholars pay more attention to the whole process of the shear stress or shear strain changing with time during the shear deformation of gelled crude oil.

When studying the rheological properties of gelled crude oil, loading conditions of constant shear stress and constant shear rate are mostly used. For the experiments under constant shear stress condition, scholars have built models to describe the relationship between the shear strain and time. But the deformation of gelled crude oil is often accompanied with structure damage. Classical models, such as the Maxwell model, Kelvin model and Burgers model, cannot describe the rheological behavior accurately. Wang, Z. [10] established a quasi-Burgers rheological model by combining the Maxwell model and the quasiKelvin model with fractional order derivatives to describe the creep behavior of gelled crude oil. Li, C.X. [11] established a nonlinear creep model which forms a creeping equation combining the nonlinear intenerating Hooken body and nonlinear hardening Newtonian fluid body by introducing intenerating and hardening variables. Liu, G. [12] introduced a damage variable which is relevant to micro-parameters to the Burgers model and built a mathematical model which can describe the viscoelastic properties of crude oil. But the fitted parameters in above models vary when the loading shear stress changes. Furthermore, calculation of the relationship between the shear strain and time under a time-dependent shear stress must be carried out through complex nonlinear superposition based on the fitted parameters obtained from constant shear stress conditions. The loading-dependent parameters and the complex nonlinear superposition limit the actual application of this kind of model in the calculation of pipeline start-up pressure.

As shown in literature [2-4], scholars often adopt the rheological model with shear rate controlled when studying the start-up of gelled crude oil pipeline. Initially, research studies mainly focused on the thixotropic process of material under constant shear rate. Thixotropic models which describe the shear stress decline of materials are divided into two categories: one is a direct expression between the shear stress and time, such as the thixotropic models proposed by literature $[13,14]$. This kind of model only applies to fitting the stress decrease under constant shear rate condition. Another model regards the structural parameter $\lambda$ as a intermediate quantity to indirectly show the changes of shear stress with time, such as the thixotropic models proposed by Cheng, D.C.H. [15], Houska, M. [16], etc. Theoretically, this kind of model can be applied to the dynamic shear rate situation. However, the two kinds of models above have not considered the shear stress increasing stage before yielding.

In fact, for most of the thixotropic materials, the shear stress increases with shear strain before yielding when being applied a constant shear rate. Therefore, scholars gradually began to pay attention to the stress increase process. A kind of thixotropic model proposed by Mujumdar, A. [17], Dullaert, K. [18] assumes that the shear stress $\sigma$ consists of the elastic stress $\sigma_{e}$ and the viscous stress $\sigma_{v}$, where $\sigma_{e}$ is associated with structural parameter $\lambda$ and strain, and $\sigma_{v}$ is a structure and shear rate dependent parameter. This kind of model can express the shear stress rising phenomenon during the initial shear deformation stage. Different from Mujumdar, A. [17] and Dullaert, K. [18], de Souza Mendes, P.R. regards the shear stress $\sigma$ as the sum of the viscous shear stress $\sigma_{v}$ and the viscoelastic shear stress $\sigma_{M}$ of a special Maxwell mechanical analog $[19,20]$. Elastic modulus $G_{M}$ and viscosity $\eta_{M}$ in the Maxwell analog are associated with structural parameter $\lambda$. When investigating the thixotropic characteristics of waxy crude oil, Teng, H. [21, 22] compared the above models and believed that the gelled crude oil with weak structure is more suitable for the kind of model proposed by Mujumdar, A. [17] and Dullaert, K. [18] instead of the one proposed by de Souza Mendes, P.R. [19, 20]. Through modifying the structure breakdown rate equation, he built a model of gelled crude oil which can accurately describe the thixotropic characteristics under dynamic shear rate conditions.

Here we regard the models proposed by Mujumdar, A. [17], Dullaert, K. [18] and Teng, H. [21, 22] as the TypeI model, and the model proposed by de Souza Mendes, P.R. $[19,20]$ as the Type-II model. The total stress of the Type-I model $\sigma$ is the sum of the elastic shear stress $\sigma_{e}$ and the viscous shear stress $\sigma_{v}$. Therefore, it can be approximately regarded as the quasi-Voigt-Kelvin mechanical ana$\log$, as shown in Figure 1a, but the elastic modulus $G$ and the viscosity $\eta$ are related to the structural parameter. The mechanical analog of the Type-II model is shown in Figure $1 \mathrm{~b}$, where $G_{M}$ and $\eta_{M}$ in the Maxwell component are 


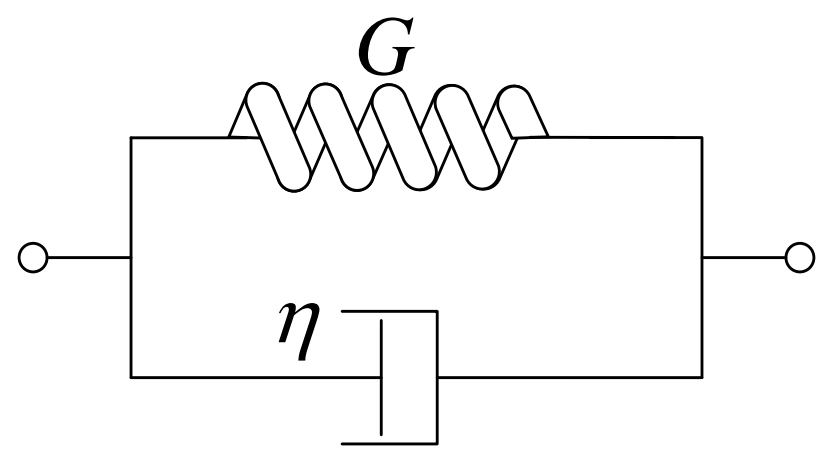

(a)

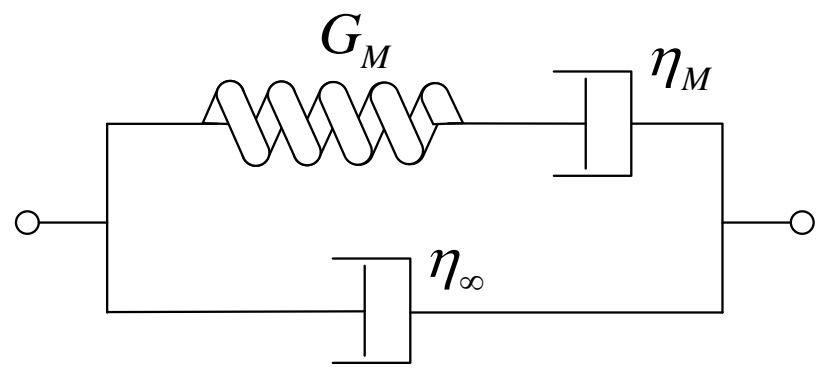

(b)

Figure 1: Mechanical analog of different models (a) Type-I model (b) Type- II model

structure-dependent parameters while $\eta_{\infty}$ is usually constant.

Similar to other viscoelastic materials, gelled crude oil shows significant creep properties under constant shear stress condition and remarkable stress relaxation properties under constant shear strain condition. Actually, the constant shear rate is a shear strain linearly increasing with time. Based on the Boltzmann superposition principle, we can find that the stress relaxation still plays an important role when calculating the shear stress under a linearly increasing shear strain. However, the Voigt-Kelvin model exhibits a strain creep but no stress relaxation [23].

In the Type-I model, the elastic stress $\sigma_{e}$ is usually determined by the elastic strain $\varepsilon_{e}$, initial shear modulus, the structural parameter, or the damping function $h\left(\varepsilon_{e}\right)$ associated with the shear strain considered in literature [21]. Within the range of initial deformation, the structural parameter almost remains unchanged and the elastic strain $\varepsilon_{e}$ is approximately equal to the total strain $\varepsilon$ of material [21]. Therefore, the elastic stress $\sigma_{e}$ is mainly determined by the shear modulus and total strain $\varepsilon$, less relevant to the shear rate $\dot{\varepsilon}$.

According to literature [17, 21], we have $\sigma_{v} \rightarrow 0$ when $\varepsilon \rightarrow 0$ or $\dot{\varepsilon} \rightarrow 0$. There will be $\sigma \approx \sigma_{e}$, which means that the material shows a significant elastic property and the total shear stress $\sigma$ is almost independent of shear rate $\dot{\varepsilon}$ when $\varepsilon \rightarrow 0$ or $\dot{\varepsilon} \rightarrow 0$. Based on the Type-I model, we can presume that during the initial deformation stage, the stressstrain curves should be similar when the loading shear rate changes under ultralow levels. An associated problem is that the total shear stress will remain large even if the loading shear rate has been reduced. The shear stresses under ultralow levels of shear rate have been forecasted based on the Type-I model in literature [21]. Result showed that there is little difference between the shear stresses under $0.0001 \mathrm{~s}^{-1}$ and $0.1 \mathrm{~s}^{-1}$. And the one under $0.0001 \mathrm{~s}^{-1}$ is even higher than the shear stresses under $0.001 \mathrm{~s}^{-1}$ and $0.01 \mathrm{~s}^{-1}$. Changing rule of the predicted shear stresses in literature [21] is significantly different from the changing rule of the measured data shown in Figure 3 and incompatible with the rules in literature [4, 5]. For pipeline restart of thixotropic drilling fluid [4] and waxy crude oil [24], velocity is almost constant in the central area of tube. The size of the low-shearing or non-shearing region is remarkable. So a robust model which is also applicable to ultralow shear rates is requried.

Theoretically, the Type-II model built by de Souza Mendes, P.R. [19] is more rigorous when compared with the Type-I model. The Maxwell mechanical analog can characterize the relaxation properties. When constant shear rate $\dot{\varepsilon}$ is applied, shear stress of the classical Maxwell analog can be described in Eq. 2. Obviously, the shear stress of Maxwell analog is related to shear rate $\dot{\varepsilon}$ and shear strain $\varepsilon$, so the defect of Type-I model is fixed. But in the model proposed by de Souza Mendes, P.R. [19], the elastic modulus $G_{M}$ and the viscosity $\eta_{M}$ in Maxwell analog are structuredependent parameters which make the model complex and difficult to fit the experimental data.

Describing the initial mechanical response and yielding behavior of gelled crude oil accurately is the precondition for calculating the start-up process of gelled crude oil pipeline. There is an urgent need for the model considering relaxation properties and fitting the experimental data easily under both normal and ultralow shear rate conditions. Inspired by the Type-II model built by de Souza Mendes, P.R. [19], we improve the classical Jeffreys mechanical analog and build a mathematical model to describe the initial mechanical response characteristics of gelled crude oil under constant shear rate. Based on the mathematical model, we propose a critical damage softening strain, which is irrelevant to loading shear rates, to describe the yielding behavior of gelled crude oil. 


\section{The Quasi-Jeffreys model}

\subsection{Maxwell mechanical analog}

As shown in Figure 2, the quasi-Jeffreys model consists of a Maxwell mechanical analog and a special dashpot in parallel. Different from the model proposed by de Souza Mendes, P.R. [19], the special dashpot in our model shows non-Newtonian properties but the elastic modulus of the spring and the viscosity of the dashpot in Maxwell analog are constant. Therefore, shear modulus of the Maxwell viscoelastic body can be described as:

$$
G(t)=G_{M} \exp \left(-\frac{G_{M}}{\eta_{M}} t\right)
$$

Where $G$ is the modulus of Maxwell mechanical analog, $\mathrm{Pa} ; G_{M}$ is the elastic modulus of the spring component in Maxwell analog, $\mathrm{Pa} ; \eta_{M}$ is the viscosity of the dashpot in Maxwell analog, $\mathrm{Pa} \cdot \mathrm{s}$ and $t$ is the time, $\mathrm{s}$.

Under a constant shear rate $\dot{\varepsilon}$, the shear stress $\sigma_{M 1}$ of Maxwell analog can be described as:

$$
\begin{aligned}
\sigma_{M 1} & =\eta_{M} \dot{\varepsilon}\left[1-\exp \left(-\frac{G_{M}}{\eta_{M}} t\right)\right] \\
& =\eta_{M} \dot{\varepsilon}\left[1-\exp \left(-\frac{G_{M}}{\eta_{M}} \frac{\varepsilon}{\dot{\varepsilon}}\right)\right]
\end{aligned}
$$

Where $\dot{\varepsilon}$ is the shear rate, $\mathrm{s}^{-1} ; \varepsilon$ is the shear strain, dimensionless; $\sigma_{M 1}$ is the shear stress of Maxwell analog, Pa.

\subsection{Special dashpot}

The special dashpot is described by the formula of powerlaw fluid of which the consistency coefficient is variable, as shown in Eq. 3.

$$
\sigma_{s 1}=K(t) \dot{\varepsilon}^{n}
$$

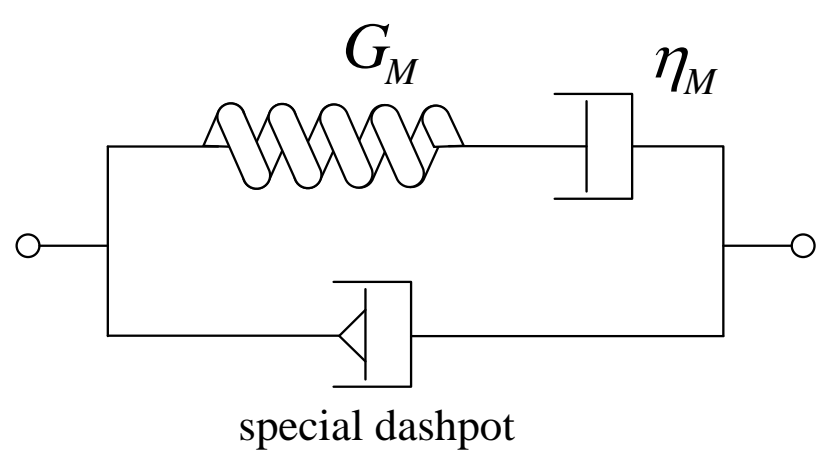

Figure 2: Diagram of the quasi-Jeffreys model
Where $\sigma_{s 1}$ is the shear stress of the special dashpot, Pa; $K$ is the consistency coefficient of the special dashpot, $\mathrm{Pa} \cdot \mathrm{s}^{n}$, and $n$ is a rheological index. As shown in the Houska model [16], $K(t)$ decreases with time under constant shear rate condition. The structural parameter $\lambda$ was used to describe $K(t)$ as

$$
K(t)=\lambda K_{0}+K_{1}
$$

Where $K_{0}$ is the structure-dependent consistency, Pa.s ${ }^{n}$; $K_{1}$ is the unstructured coefficient, $\mathrm{Pa} \cdot \mathrm{s}^{n}$, and $\lambda$ is the structural parameter ranging from 0 to 1 . Kinetic equation of the structural parameter is:

$$
\frac{\mathrm{d} \lambda}{\mathrm{d} t}=a(1-\lambda)-b \lambda \dot{\varepsilon}^{m}
$$

Where $a$ is a kinetic constant for structure buildup, $b$ is a kinetic constant for shear-induced breakdown, and $m$ is a dimensionless constant. During the initial shear deformation stage of gelled crude oil, there is no significant structure breakdown. And there will be $\lambda \rightarrow 1$ and $1-\lambda \rightarrow 0$. Therefore, the structure buildup can be neglected. Then Eq. 5 can be transformed to:

$$
\frac{\mathrm{d} \lambda}{\mathrm{d} t}=-b \lambda \dot{\varepsilon}^{m}
$$

Integration was applied to Eq. 6,

$$
\ln \lambda=\int-b \dot{\varepsilon}^{m} \mathrm{~d} t
$$

Approximation was applied to the right-hand side of Eq. 7. The integral function which is relevant to the shear rate and time can be described as a strain-dependent function:

$$
\int-b \dot{\varepsilon}^{m} \mathrm{~d} t \approx-b^{\prime}\left(\int \dot{\varepsilon} \mathrm{d} t\right)^{m^{\prime}}=-b^{\prime} \varepsilon^{m^{\prime}}+C
$$

For the convenience of writing, we still use $b$ and $m$ to replace $b^{\prime}$ and $m^{\prime}$ in Eq. 8. So, at the initial shear deformation stage, the structural parameter $\lambda$ can be approximately described as

$$
\ln \lambda=-b \varepsilon^{m}+C
$$

When $\varepsilon=0$, we have $\lambda=1$ and $C=0$. Eq. 9 can be transformed to:

$$
\lambda=\exp \left(-b \varepsilon^{m}\right)
$$

For the thixotropic crude oil, shear stress usually decreases significantly after yielding. Compared with structure-dependent consistency $K_{0}$, the value of $K_{1}$ is too negligible. During the initial shear deformation stage, we convert Eq. 4 as:

$$
K(t)=K_{0} \exp \left(-b \varepsilon^{m}\right)
$$




\subsection{Total shear stress}

\subsubsection{Introduction of damage variable}

Damage mechanics aims to quantitatively represent the accrual of mechanical deterioration of a material component subjected to a certain loading. This is done by introducing a damage variable. Liu, G. [12] and Li, C.X. [11] introduced damage variable into waxy crude oil to describe the whole process of the structure breakdown. In this article, we argue that the damage variable is used to describe the mechanics when the precipitated 3-D network of waxy crystals still keeps continuous. That is the initial deformation stage before significant structure breakdown. Then the structural parameter was only determined by kinetic equation when waxy crystals become scattered during the significant decay stage of consistency. In other words, the damage variable is used to characterize the transition of gelled crude oil from viscoelastic to viscous.

Besides, Liu, G. [12] and Li, C.X. [11] considered the damage variable as a function of time. Considering that scholars $[8,9]$ choose the shear strain as the index to measure the yielding of gelled crude oil, we regard the damage variable of gelled crude oil as a function of the shear strain. The Weibull distribution is widely used to describe the failure characteristics of materials $[25,26]$. Here, a straindependent Weibull distribution can be expressed as:

$$
f(\varepsilon)=\frac{k}{\delta}\left(\frac{\varepsilon}{\delta}\right)^{k-1} \exp \left[-\left(\frac{\varepsilon}{\delta}\right)^{k}\right]
$$

Where $k$ represents the shape parameter; $\delta$ represents the scale parameter; $f(\varepsilon)$ is the partial derivative of the damage with respect to the shear strain. The evolution of damage can be defined as a cumulative distribution function for the Weibull distribution:

$$
\frac{\mathrm{d} D(\varepsilon)}{\mathrm{d} \varepsilon}=f(\varepsilon)
$$

So the damage variable can be expressed as a function of shear strain:

$$
D(\varepsilon)=1-\exp \left[-\left(\frac{\varepsilon}{\delta}\right)^{k}\right]
$$

When $\varepsilon=0$, we get $D(\varepsilon)=0,1-D(\varepsilon)=1$. It means that no damage occurs. When $\varepsilon \rightarrow+\infty$, there will be $D(\varepsilon)=1$ and $1-D(\varepsilon)=0$. The material is completely damaged. Here, $1-D(\varepsilon)$ can be regarded as a coefficient characterizing the integrity of the 3-D network of waxy crystals.

\subsubsection{Final shear stress expression considering damage}

During the initial deformation stage, viscous properties of gelled crude oil are less obvious, because the built 3-D network structure of waxy crystals can trap the liquid phase inside the crude oil [27]. With the increase of strain, liquid oil will be released gradually due to the structural damage of crystals and the viscous properties will be more significant. So the shear stress of the special dashpot in Figure 2 is modified by introducing damage variable $D(\varepsilon)$ to Eq. 3 and expressed as:

$$
\sigma_{s}=D(\varepsilon) \sigma_{s 1}=D(\varepsilon) K(t) \dot{\varepsilon}^{n}
$$

Where $\sigma_{s}$ is the modified shear stress of the special dashpot considering structural damage, Pa. Meanwhile, the Maxwell body losses its viscoelastic properties gradually due to the structural damage. Therefore, we introduce the integrity characteristic coefficient $1-D(\varepsilon)$ to Eq. 2 . Then the shear stress of the Maxwell analog under constant shear rate condition is expressed as:

$$
\begin{aligned}
\sigma_{M} & =[1-D(\varepsilon)] \sigma_{M 1} \\
& =[1-D(\varepsilon)] \eta_{M} \dot{\varepsilon}\left[1-\exp \left(-\frac{G_{M}}{\eta_{M}} \frac{\varepsilon}{\dot{\varepsilon}}\right)\right]
\end{aligned}
$$

Where $\sigma_{M}$ is the shear stress of the Maxwell body in which the integrity characteristic coefficient is introduced. Under the condition of constant shear rate, the total shear stress $\sigma$ can be described as:

$$
\begin{aligned}
\sigma & =\sigma_{s}+\sigma_{M} \\
& =[1-D(\varepsilon)] \eta_{M} \dot{\varepsilon}\left[1-\exp \left(-\frac{G_{M}}{\eta_{M}} \frac{\varepsilon}{\dot{\varepsilon}}\right)\right]+D(\varepsilon) K(t) \dot{\varepsilon}^{n}
\end{aligned}
$$

Introduce Eq. 14 into Eq. 17,the expression of total shear stress can be obtained as Eq. 18:

$$
\begin{aligned}
\sigma & =\eta_{M} \dot{\varepsilon}\left[1-\exp \left(-\frac{G_{M}}{\eta_{M}} \frac{\varepsilon}{\dot{\varepsilon}}\right)\right] \exp \left[-\left(\frac{\varepsilon}{\delta}\right)^{k}\right] \\
& +\left\{1-\exp \left[-\left(\frac{\varepsilon}{\delta}\right)^{k}\right]\right\} K_{0} \exp \left(-b \varepsilon^{m}\right) \dot{\varepsilon}^{n}
\end{aligned}
$$

Chang, C. [7] argued that the yielding of waxy crude oil occurs by an initial elastic response, followed by viscoelastic creep and a final fracture. Based on the quasi-Jeffreys model, we can find that $D(\varepsilon) \rightarrow 0$ when $\varepsilon \rightarrow 0$. So, the model can be simplified as a Maxwell body when $\varepsilon \rightarrow 0$. Therefore, the quasi-Jeffreys model shows an elastic property during the initial deformation stage as the Maxwell model does. As the shear strain increases, $D(\varepsilon)$ will increase and the effect of special dashpot will be significant, so the quasi-Jeffreys model will show a creep property due to the viscous effect of the special dashpot. When 
Table 1: Basic properties of two crude oil samples

\begin{tabular}{cccc}
\hline Oil samples & wax precipitation point $\left({ }^{\circ} \mathrm{C}\right)$ & condensation point $\left({ }^{\circ} \mathrm{C}\right)$ & Measuring temperature $\left({ }^{\circ} \mathrm{C}\right)$ \\
\hline GNPOC crude oil & 54 & 33 & 25 \\
Nanyang crude oil & 62 & 47 & 45 \\
\hline
\end{tabular}

$D(\varepsilon) \rightarrow 1$, the shear stress of Maxwell body $\sigma_{M} \rightarrow 0$, the quasi-Jeffreys model will show a final fracture due to the thixotropic consistency of the special dashpot. So the quasi-Jeffreys model can describe the whole yielding process of gelled crude oil theoretically.

Under constant shear strain conditions, shear rate $\dot{\varepsilon}=$ 0 , and then $\sigma_{s}=0$. The quasi-Jeffreys model can be regarded as the Maxwell model and exhibits a significant stress relaxation. This is a remarkable advance by contrast with the Type-I model.

\section{Experimental data fitting}

Experiments under constant shear rate conditions were conducted using GNPOC crude oil and Nanyang crude oil. Basic properties of the two oil samples are shown in Table 1.

Anton Paar Physica MCR301 rheometer was used to carry out the experiments. Measuring system of the rheometer is coaxial cylinder apparatus CC27. The minimum angular velocity of the instrument is $10^{-9} \mathrm{rad} / \mathrm{s}$ and the corresponding shear rate is about $1.25 \times 10^{-8} \mathrm{~s}^{-1}$. The experiments were carried out as follows. First, the oil temperature was reduced from $70^{\circ} \mathrm{C}$ to the measuring temperature with a cooling rate of $0.2^{\circ} \mathrm{C} / \mathrm{min}$ under a constant shear rate of $60 \mathrm{~s}^{-1}$. Then the oil sample was kept static for 2 hours under the constant temperature condition to make sure that the gelation was fully formed. Finally, the mechanical response of gelled crude oil was tested under constant shear rate conditions. The experimental results were shown in Figure 3.

From Figure 3 we can get that the shear stress is always smaller under lower shear rate which is consistent with the experimental data in literature $[4,5]$ and different from the model prediction in literature [21]. From both Figure $3 a$ and Figure $3 \mathrm{~b}$, we can see that the shear stress decreases with the shear strain when the shear strain exceeds a certain value even under the shear rate of $0.0001 \mathrm{~s}^{-1}$. That is, we get $\mathrm{d} \lambda / \mathrm{d} t<0$ even the shear rate $\dot{\varepsilon} \rightarrow 0$. Combined with Eq. 5,we can get $a(1-\lambda)<b \lambda \dot{\varepsilon}^{m} \rightarrow 0$. So we can believed that the structure buildup can be neglected during the ini-

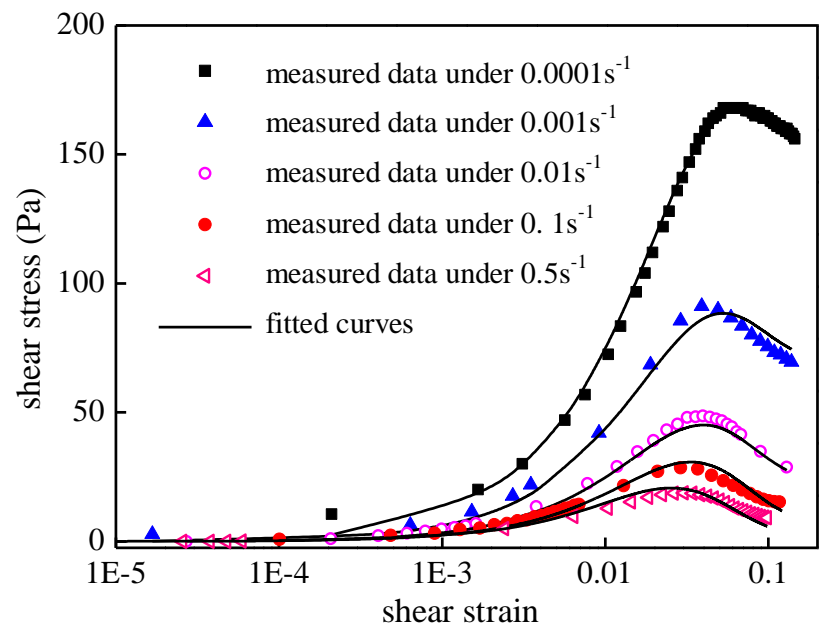

(a)

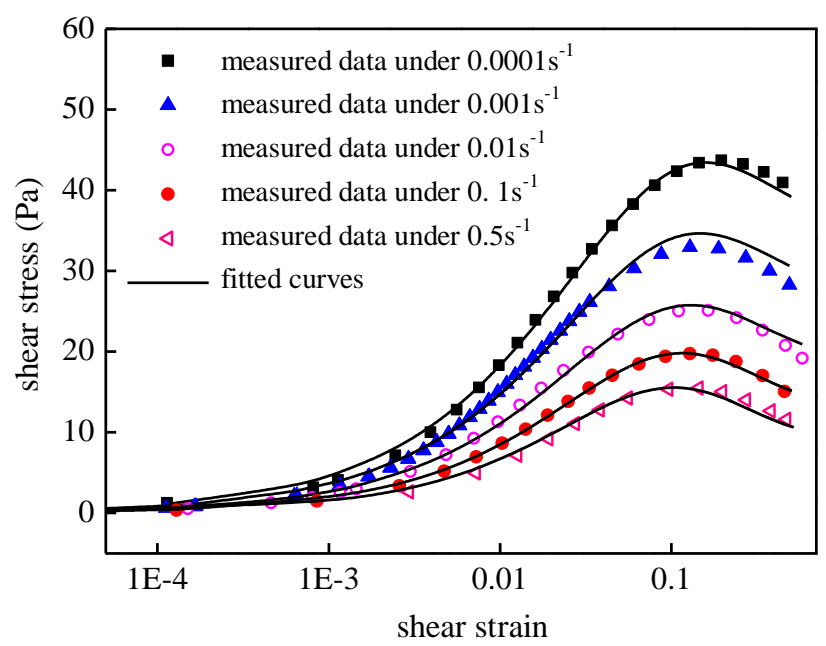

(b)

Figure 3: Experimental data and fitting curves (a) GNPOC crude oil (b) Nanyang crude oil

tial shear deformation stage, which verifies the hypothesis in seeking Eq. 6 from Eq. 5.

$3 \mathrm{D}$ graphics of the experimental data was established by regarding the shear strain as $x$-axis, the shear rate as $y$ axis and the shear stress as $z$-axis, so that the experimental data can be fitted by Eq. 18 directly through the software MATLAB. Fitted parameters in Eq. 18 are shown in Table 2. 
Table 2: Fitted parameters in the quasi-Jeffreys model

\begin{tabular}{ccccccccc}
\hline parameters & $\eta_{M}(\mathrm{~Pa} \cdot \mathrm{s})$ & $G_{M}(\mathrm{~Pa})$ & $\delta(-)$ & $k(-)$ & $K_{0}\left(\mathrm{~Pa} \cdot \mathrm{s}^{n}\right)$ & $b(-)$ & $m(-)$ & $n(-)$ \\
\hline GNPOC crude oil & $9.404 \times 10^{5}$ & 2131.887 & $3.149 \times 10^{-2}$ & 1.023 & 537.801 & 1.348 & 0.188 & 0.474 \\
Nanyang crude oil & $9.316 \times 10^{6}$ & 406.455 & $3.996 \times 10^{-2}$ & 0.657 & 60.290 & 0.402 & 0.237 & 0.160 \\
\hline
\end{tabular}

As shown in Figure 3, Eq. 18 fits the experimental data well even the shear rate varies from $0.0001 \mathrm{~s}^{-1}$ to $0.5 \mathrm{~s}^{-1}$. Different from the models proposed by Teng, H. [21] and de Souza Mendes, P.R. [19], the quasi-Jeffreys model in this article focuses on the mechanics of the initial shear deformation stage rather than the whole structural breakdown procedure after yielding. The kinetic equation of structural parameter in differential form is avoided by using Eq. 18 so that data fitting can be conducted by means of software directly and easily.

Based on Table 2, we can get $\eta_{M} / G_{M}=441.112$ for GNPOC crude oil and $\eta_{M} / G_{M}=23030.840$ for Nanyang crude oil. For the Maxwell model, $\eta_{M} / G_{M}$ is a characteristic time, describing the relaxation behavior. The relaxation time will be longer and the elastic property of material will be more significant when $\eta_{M} / G_{M}$ increases. When $\eta_{M} / G_{M} \rightarrow+\infty$, the Maxwell model in Figure 2 can be simplified as an elastic model. And the proposed quasiJeffreys model can be reduced to the Type-I model.

Based on the value of $\eta_{M} / G_{M}$, we can see that the relaxation property of GNPOC crude oil is more significant but Nanyang crude oil shows more elastic property. This is consistent with the experimental data in Figure 3. The maximum shear stress of GNPOC crude oil under different shear rates varies from about 20Pa to 170Pa while Nanyang crude oil's changes from about $10 \mathrm{~Pa}$ to $50 \mathrm{~Pa}$. That is, the shear stress of GNPOC crude oil shows more "shear rate dependent" and "viscous", but Nanyang crude oil shows more "shear strain dependent" and "elastic". So the quasiJeffreys model is more suitable for GNPOC crude oil.

\section{Results and discussion}

\subsection{The changing law of $\sigma_{M}$ and $\sigma_{s}$ during the initial deformation stage}

The quasi-Jeffreys model in this article argues that the shear stress of gelled crude oil consists of the shear stress of Maxwell viscoelastic body, $\sigma_{M}$ and the shear stress of special dashpot, $\sigma_{s}$. Further research on the mechanics of gelled crude oil during the initial shear deformation stage is carried out by analyzing the changing law of $\sigma_{M}$ and $\sigma_{s}$.
Considering that the relaxation properties of GNPOC crude oil is more significant, the following discussion will be carried out based on the data of GNPOC crude oil. According to Eq. 18 and Table 2, the changing laws of $\sigma_{s}$ in Eq. 15 and $\sigma_{M}$ in Eq. 16 of GNPOC crude oil under different shear conditions were calculated, as shown in Figure 4.

From Figure 4 we can see that $\sigma_{M}$ increases first and then decreases with the shear strain during the initial shear deformation stage. $\sigma_{s}$ also increases with the shear strain but there is no significant decrease. Based on Eq. 2, we know that the shear stress of the classical Maxwell ana$\log$ increases with the shear strain gradually under constant shear rate conditions. But based on Eq. 16 in which damage variable has been introduced, we can explain that the shear stress will decrease significantly when the shear strain increases beyond a certain range. From the changing law of $\sigma_{s}$, we know that the viscous properties of gelled crude oil become significant because the participated 3-D network structure of waxy crystals damages. But the structure breakdown is not remarkable in the initial shear strain range.

Under high shear rates, such as $0.1 \mathrm{~s}^{-1}$ and $0.5 \mathrm{~s}^{-1}, \sigma_{M}$ is always less than $\sigma_{s}$ during the initial shear deformation stage.The proportion of $\sigma_{M}$ to the total shear stress increases when the loading shear rate is reduced. On the contrary, the special dashpot gradually takes the leading role when the loading shear rate is enhanced.

The shear strain at the position of the maximum total shear stress, which is defined as the critical yield strain, is slightly different under different shear rates, and the critical yield strain tends to be higher when the shear rate is enhanced. This is consistent with the experimental data in Figure 3 and Figure 4.

$\varepsilon_{C M}$ was defined as the shear strain at the position of the maximum $\sigma_{M}$. If the Maxwell analog is suddenly subjected to a shear stress or a shear strain, the elastic element will deform at once and the viscous element deform with time gradually [23]. The shorter the deformation time is, the less viscous characteristics but more elastic properties will be shown. Under higher shear rate conditions, the time needed for the shear strain to reach $\varepsilon_{C M}$ becomes shorter. The Maxwell body behaves like an elastic material and $\sigma_{M}$ becomes almost exclusively related to shear strain $\varepsilon$. Curves of the relationship between $\sigma_{M}$ and $\varepsilon$ will be the 

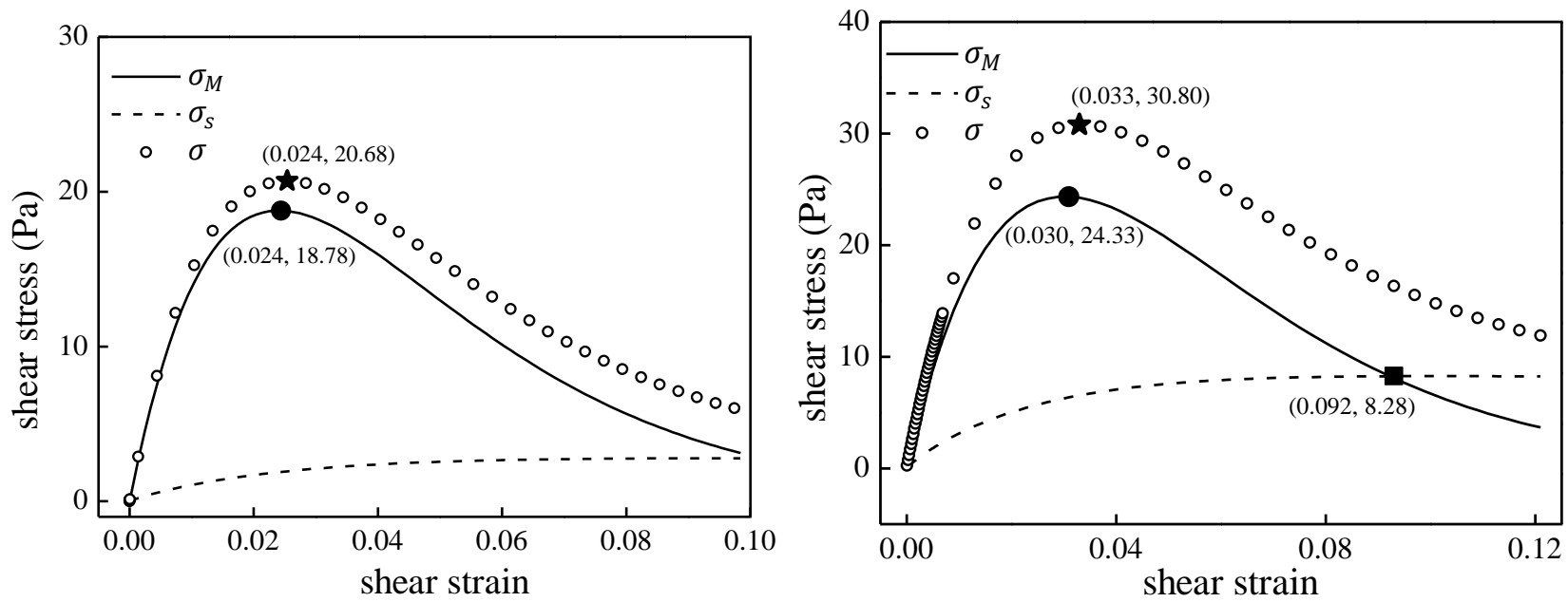

(a)

(b)
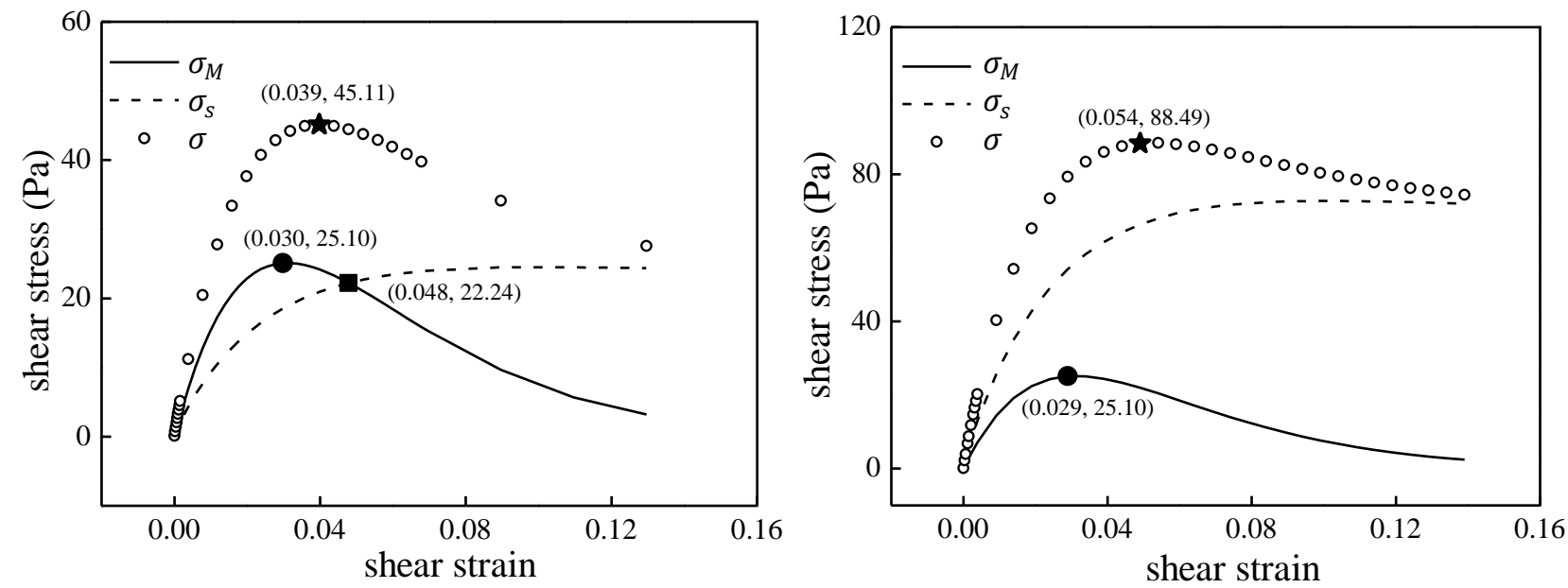

(c)

(d)

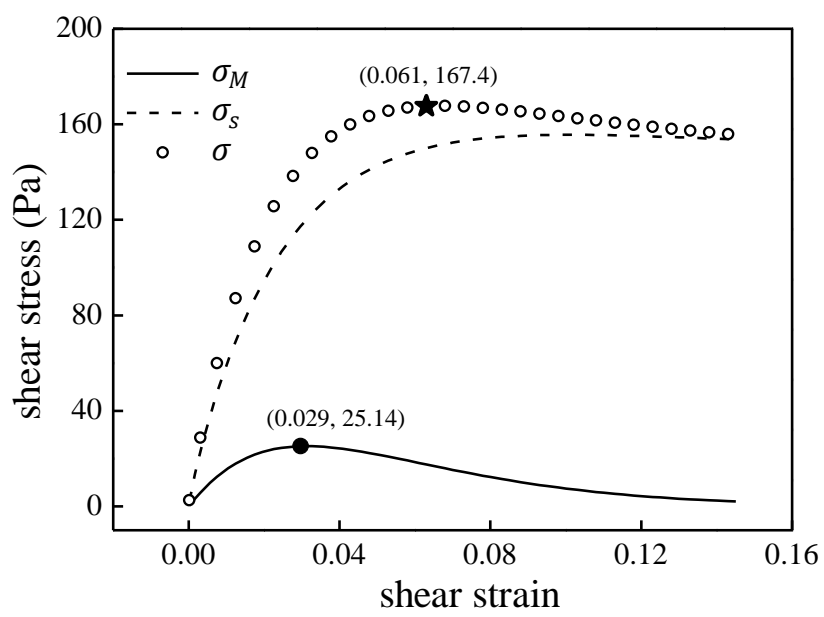

(e)

Figure 4: Calculation of $\sigma_{M}, \sigma_{s}$ and total shear stress $\sigma$ at the initial deformation stage under different shear rates (a) $0.0001 \mathrm{~s}^{-1}$ (b) $0.001 \mathrm{~s}^{-1}$ (c) $0.01 \mathrm{~s}^{-1}$ (d) $0.1 \mathrm{~s}^{-1}$ (e) $0.5 \mathrm{~s}^{-1}$ (In this figure, $\bullet$ represents the maximum of $\sigma_{M}$, $\star$ represents the maximum of total shear stress $\sigma$, represents that $\sigma_{M}=\sigma_{s}$ ) 
same under different shear rate conditions. So from Figure 4, we can find that $\varepsilon_{C M}$ varies little under high shear rate conditions $\left(0.001^{-1}\right.$ and above). But under low shear rate conditions, such as $0.0001^{-1}, \varepsilon_{C M}$ and $\sigma_{M}$ are significantly lower than those under other shear rate conditions.

\subsection{Critical damage softening strain}

During the yielding process of gelled crude oil, the critical yield strain under different loading conditions are approximately the same, scholars often regard the critical yield strain as a reasonable parameter to indicate the yielding behavior and reflect the actual properties of gelled crude oil. Actually, the critical yield strain is determined by the changing law of the total shear stress, and the total shear stress is related to the shear rate closely. So the critical yield strain can't be independent of the shear rate completely. According to the above research, we definitely found that the critical yield strains under different shear rates are slightly different. Compared with the critical yield strain, $\varepsilon_{C M}$ keeps constant under high shear rate conditions but still changes under low shear rates, because $\sigma_{M}$ is still related to the shear rates.

These two critical strains defined by the changing rules of shear stress still can't be independent of the shear rate completely. In this section, a damage variable $D(\varepsilon)$ was introduced to characterize the softening process from the viscoelastic to viscous. Compared with the shear stress, $D(\varepsilon)$ is only relevant to the shear strain $\varepsilon$. Here, we define the critical damage softening strain $\varepsilon_{D}$ as

$$
D\left(\varepsilon_{D}\right)=1-D\left(\varepsilon_{D}\right)=\frac{1}{2}
$$

Based on the parameters in Table 2, $D(\varepsilon)$ and $1-D(\varepsilon)$ are calculated out by using Eq. 14, as shown in Figure 5 .

From Figure 5, we can find that the critical damage softening strain $\varepsilon_{D}$ remains uniform under different shear rates for a specific crude oil sample. For this two waxy crude oil samples, $\varepsilon_{D}$ is smaller than the critical yield strain under the experimental temperature. Different from the critical yield strain, the value of the critical damage softening strain $\varepsilon_{D}$ can't be obtained directly through the changing law of total shear stress. $\varepsilon_{D}$ should be calculated by the parameters of the quasi-Jeffreys model which are obtained from the experimental data under different loading shear rates. So $\varepsilon_{D}$ is a common parameter under different shear conditions and can reflect the true properties of gelled crude oil appropriately.

But what happens actually inside the crude oil when the shear strain reaches $\varepsilon_{D}$ is still unknown. Further research on the microscopic parameters of waxy crystals at

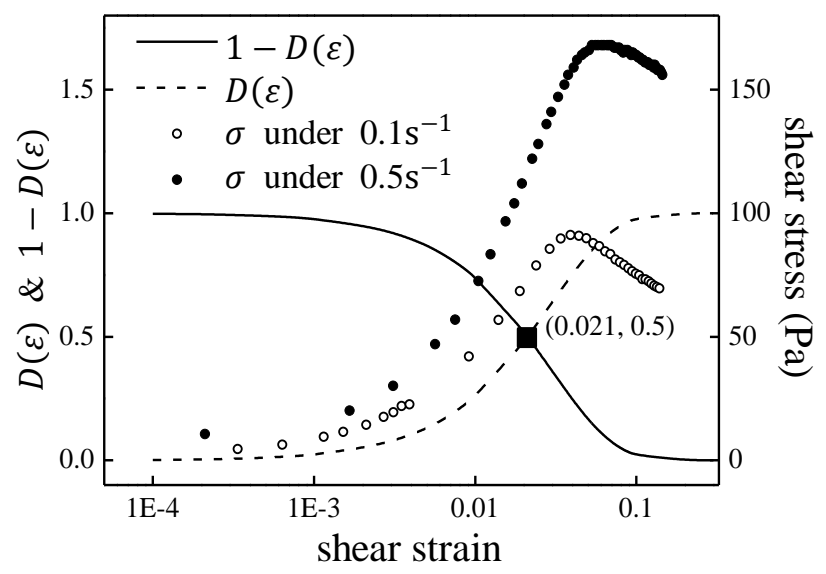

(a)

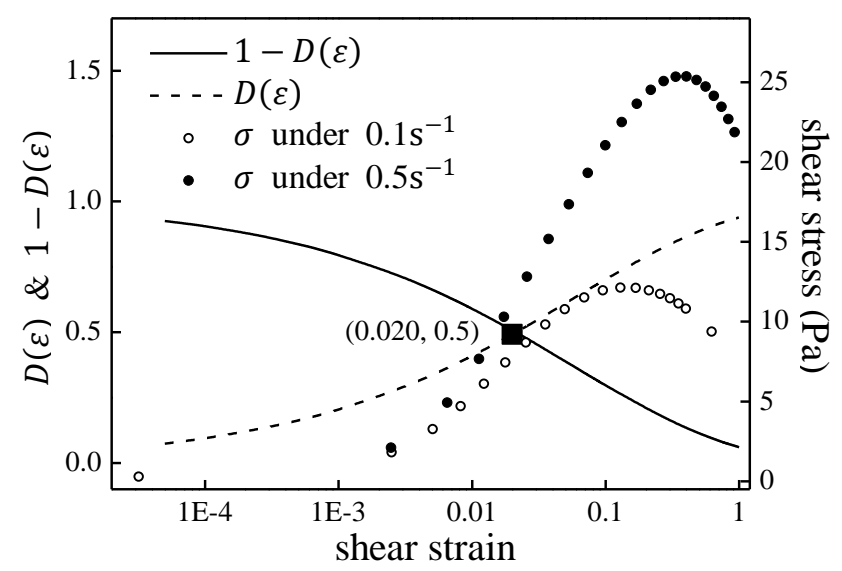

(b)

Figure 5: Damage variable and total shear stress under different shear rates (a) GNPOC crude oil (b) Nanyang crude oil ( $\square$ signifies the point where $D(\varepsilon)=1-D(\varepsilon)$ )

the critical strain $\varepsilon_{D}$ and the changing rules of $\varepsilon_{D}$ with paraffin content or temperature is worth being conducted.

\section{Conclusions}

1. The quasi-Jeffreys model was proposed by putting the Maxwell mechanic analog and the special dashpot in parallel. Constitutive equation of the quasiJeffreys was obtained by introducing the damage variable to the constitutive equations of the Maxwell mechanic analog and the special dashpot. Using the quasi-Jeffreys model to fit the experimental data under different shear rate conditions, we can find that the model can fit the experimental data under the shear rates from $0.0001 \mathrm{~s}^{-1}$ to $0.5 \mathrm{~s}^{-1}$. 
2. During the initial shear deformation stage, $\sigma_{M}$ increases first and then decreases with the shear strain and $\sigma_{s}$ also increases with the shear strain but there is no significant decrease. The proportion of $\sigma_{M}$ to the total shear stress increases when the shear rate is reduced but $\sigma_{s}$ changes oppositely. The critical yield strain and $\varepsilon_{C M}$ still change slightly under different shear rates.

3. The critical damage softening strain $\varepsilon_{D}$ could be regarded as a better parameter to reflect the true properties of gelled crude oil.

Acknowledgement: This work is supported by National Natural Science Foundation of China (51704319), (51574274) and Project Funded by China Postdoctoral Science Foundation (2016M602225).

\section{References}

[1] Chang C., Nguyen Q.D., Rřnningsen H.P., Isothermal startup of pipeline transporting waxy crude oil, J. Nonnewton. Fluid Mech., 1999, 87, 127-154. doi:10.1016/S0377-0257(99)00059-2

[2] Wachs A., Vinay G., Frigaard I., A 1.5D numerical model for the start up of weakly compressible flow of a viscoplastic and thixotropic fluid in pipelines, J. Nonnewton. Fluid Mech., 2009, 159, 81-94. doi:10.1016/j.jnnfm.2009.02.002

[3] Zhang G., Xiao W., Liu G., Lan H., The Initial Startup Wave Velocity in Isothermal Pipeline With Compressible Gelled Crude Oil, SPE J., 2014, 19, 418-424.

[4] Negrăo C.O.R., Franco A.T., Rocha L.L.V., A weakly compressible flow model for the restart of thixotropic drilling fluids, J. Nonnewton. Fluid Mech., 2011, 166, 1369-1381. DOI:10.1016/j.jnnfm.2011.09.001

[5] Lan H., Zhang Z.G., Liu G., Wang W.Q., Lu X.G., Mechanical behavior of gelled crude oil under constant shear rate in initial structural failure process, J. China Univ. Pet. (Edition Nat. Sci.), 2009, 33, 117-121.

[6] Japper-Jaafar A., Bhaskoro P.T., Sean L.L., Sariman M.Z., Nugroho H., Yield stress measurement of gelled waxy crude oil: Gap size requirement, J. Nonnewton. Fluid Mech., 2015, 218, 7182.

[7] Chang C., Boger D. V, Nguyen Q.D., The yielding of waxy crude oils, Ind. Eng. Chem. Res., 1998, 37, 1551-1559. DOI:10.1021/ie970588r

[8] Li C.X., Li Q.G., Research on yielding behavior of Xinjiang gelled crude oil, Oil Gas Storage and Transp., 1999, 18, 5-7.
[9] Hou L., Experimental study on yield behavior of Daqing crude Oil, Rheol. Acta., 2012, 51, 603-607.

[10] Wang Z., Zhang G., Liu G., A description of rheological model for gelled crude oil using fractional order derivatives, J. China Univ. Pet. (Edition Nat. Sci.), 2008, 32, 114-118.

[11] Li C.X., Lin M.Z., Yang F., Ma Y., A nonlinear creep model of gelled waxy crude oil, J. Chem. Eng. Chinese Univ., 2011, 25, 579- 583.

[12] Liu G., Lu X., Xu B., Zhang G., A viscoelastic/plastic damage model for gelled crude oils, Acta Pet. Sin., 2011, 32, 1078-1082.

[13] Zhang Z.B., Zhang G.Z., Thixotropy of Waxy Oil Flowing Through Pipeline, J. China Univ. Pet. (Edition Nat. Sci.), 2001, 25, 72-74.

[14] Dong P.S., Zhang G.Z., Hu X.J., A Novel Thixotropy Model for Waxy Crude Oil, Oil Gas Storageand Transp., 2005, 24, 29-32.

[15] Cheng D.C.H., Yield stress: A time-dependent property and how to measure it, Rheol. Acta., 1986, 25, 542-554. DOI:10.1007/BF01774406

[16] Houska M., 1981, Engineering aspects of the rheology of thixotropic liquids (PhD thesis), Prague, Czech Technical University

[17] Mujumdar A., Beris A.N., Metzner A.B., Transient phenomena in thixotropic systems, J. Nonnewton. Fluid Mech., 2002, 102, 157178. DOI:10.1016/S0377-0257(01)00176-8

[18] Dullaert K., Mewis J., A structural kinetics model for thixotropy. J. Nonnewton. Fluid Mech., 2006, 139, 21-30. DOI:10.1016/j.jnnfm.2006.06.002

[19] de Souza Mendes P.R., Thixotropic elasto-viscoplastic model for structured fluids, Soft Matter., 2011, 7, 6:2471-2483. DOI:10.1039/c0sm01021a

[20] de Souza Mendes P.R., Thompson R.L., A unified approach to model elasto-viscoplastic thixotropic yield-stress materials and apparent yield-stress fluids, Rheol. Acta ., 2013, 52, 673-694.

[21] Teng H., Zhang J., Modeling the Thixotropic Behavior of Waxy Crude, Ind. Eng. Chem. Res., 2013, 52, 8079-8089. DOI:10.1021/ie400983e

[22] Teng H., Zhang J., Modeling the viscoelasto-plastic behavior of waxy crude, Pet. Sci., 2013, 10, 395-401.

[23] Mainardi F., Spada G., Creep, relaxation and viscosity properties for basic fractional models in rheology, Eur. Phys. J. Spec. Top., 2011, 193, 133-160. DOI:10.1140/epjst/e2011-01387-1

[24] Vinay G., Wachs A., Agassant J.-F., Numerical simulation of weakly compressible Bingham flows: the restart of pipeline flows of waxy crude oils, J. Nonnewton. Fluid Mech., 2006, 136, 93-105.

[25] Zheng J.L., Lu S.T., Tian X.G., Viscoelastic damage characteristics of asphalt based on creep test, Eng. Mech., 2008, 25, 193196.

[26] Papoulis A., Pillai S.U., 2002, Probability, random variables, and stochastic processes (4th ed.), New York, Tata McGraw-Hill Education.

[27] Singh P., Venkatesan R., Fogler H.S., Nagarajan N., Formation and aging of incipient thin film wax-oil gels, AIChE J., 2000, 46, 1059-1074. DOI:10.1002/aic.690460517. 